Papers and Proceedings of the Royal Society of Tasmania, Volume 110, 1976

(ms. received 26.9 .1975 )

\title{
ADDITIONS TO THE LICHEN FLORA OF TASMANIA II
}

by G.C. Bratt, University of 'asmania.

and J.A. Cashin, 73 Windsor St, , Glenorchy

\section{ABSTRACT}

New lichen records for Tasmania from recent collections (31), collections of W.A. Weymouth (39) and publications (31) are made. The total number of lichen species in Tasmania is estimated to be 460 . Numerical comparisons with other areas are made and Victoria is shown to have the greatest floral affinity with Tasmania.

\section{INTRODUCTION}

The lichen flora of Tasmania has previously been extended (Bratt and Cashin 1975) by 35 taxa from that published by Wetmore (1963) as 521 taxa. The present work gives a further extension of the Tasmanian records from three sources viz. -

(a) 31 taxa collected by the present authors, details of which are given in Table 1 .

(b) 39 taxa collected by W.A. Weymouth in the period 1891-1910 which are housed at the Herbarium of the University of Tasmania. Details of taxa, other than those already mentioned by Wetmore are listed in Table 2 .

(c) 31 taxa recorded in recent 1iterature or publications overlooked by Wetmore the details of which are set out in Table 3 .

Although Wetmore lists 521 taxa the present authors have estimated that by taking synonyms, misidentification, and trivial indistinguishable forms into account there are 324 valid taxa in the list. Hence it is presently estimated there are 460 taxa validly recorded in Tasmania, a number revisable downwards as nomenclatural problems are examined and upwards as a large number of crustose collections are identified. Numerical comparisons of floras via species density (Wetmore 1967) and Sörenson coefficients (Sheard 1962) have been made and are listed in Table 4.

The numerical comparisons are crude and are made cruder by difficulties in nomenclature and the poor recording in some areas. It is expected that comparisons between Tasmania and other Australian States will be improved when unpublished work by various Australian authors becomes available. below.

Some interesting points emerge from the data of Table 4 and these are summarised

(a) For regions with areas of a similar magnitude (e.g. Tasmania, Victoria, New Zealand and Tenessee) the species density is also of similar magnitude. Since the N.Z. 1ichen flora is reasonably well established it is suggested that the closeness of the species density values for New Zealand and Tasmania indicates that a large proportion of the Tasmanian species have been recorded. The low species density values for regions such as Queensland which has been fairly well investigated (Shirley 1888, 1889) may be due to the relatively small climatic diversity of such a large region.

(b) The Sörenson coefficients show as expected that the closest geographical areas, Victoria and New South wales have the closest floral affinities with Tasmania. The rather strong resemblance between Tasmanian and New Zealand floras is also apparent. (c) The rather high value of the Sörenson coefficient for Tasmania and Tennessee 
probably illustrates that in preliminary compilations of floras there are a large number of ubiquitous lichen species.

(d) The relatively high Sörenson coefficients for Victoria and Queensland compared with other Australian States may not result from climatological relationships but may reflect the extensive work in these States and Tasmania by Wilson (1892) and Shirley (1888, 1889) leading to marked increases in the number of species in common.

\section{ACKNOWLEDGEMENTS}

The assistance of a number of specialists particularly Mr. P.W. James and Dr. W.A. Weber for identification and confirmation is gratefully acknowledged.

\section{REFERENCES}

Blackman, A.J. Bratt, G.C., and Cashin, J.A., 1974 : Umbilicariaceae in Tasmania The Lichenologist, 6, 112-114.

Bratt, G.C., Blackman, A.J. and Cashin, J.A. in press : Anzia in Tasmania. The Lichenologist.

Bratt, G.C., and Cashin, J.A. 1975 : Additions to the Tasmanian lichen flora. I. Pap. Proc. R. Soc. Tasm., 109, $17-20$.

Cribb, A.B., 1954 : The algal vegetation of Port Arthur, Tasmania. Pap. Proe. R. Soc. Tasm., 88, $1-44$.

Degelius, G., 1974: The lichen genus Collema with special reference to the extra-European Species. Symbolae Botanicae Upsaliensis, $X(2), 1-215$.

Dodge, C.W., 1948: Lichens and 1ichen parasites. BANZARE Reports, Series B. Vol. VII, BANZAR Expedition Committee.

Filson, R.B., 1969 : A review of the genera Teloschistes and Xanthoria in the lichen family Teloschistaceae in Australia. MueZleria, 2 (1), 65 - 115. , 1970 : Studies in Australian lichens I. Victorian Naturalist $\underline{87}(11), \quad 324-327$.

Henssen, A., 1969 : Three non-marine species of the genus Lichina. The Lichenologist, $4,88-98$.

Kurokawa, S., 1969 : A note on some rare lichens in Japan. J. Jap. Bot., 44 (8), $225-229$.

Kurokawa, S., Elix, J.A., Watson, P.L., and Sargent, M.V. 1971 : Parme $2 i a$ notata, A new 1 ichen species producing two new depsidones. J. Jap. Bot., 46 (2), 33 - 36.

Martin, W., 1965 (a) : Comparison of the lichen floras of New Zealand and Tasmania. Trans. R. Soc. N.Z., 3 (1), $1-6$. ., 1965 (b) : The lichen genus CZadia. Trans. R. Soc. N.Z., 3 (2), $7-12$. , 1966 : Census Catalogue of the Lichen Flora of New Zealand. Trans. R. Soc. N.Z., 3(8), $139-159$.

., 1968 : Supplement to census catalogue of New Zealand lichens.

Wrans. R. Soc. N.Z., 3 (13), $203-208$. 
Sheard, J.W. 1962 : A contribution to the lichen flora of Jan Mayen. The Lichenologist, 2 (1), $76-85$.

Shirley, J. 1888 : The lichen flora of Queensland. Proc. R. Soc. Qd., 5, $80-110$.

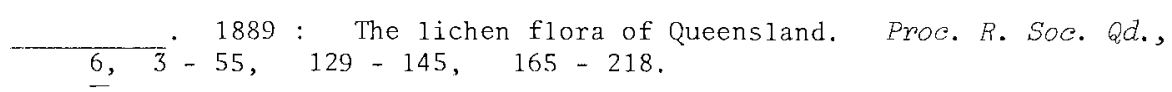

Skorepa, A.C., 1972 : A catalogue of the lichens reported from Tennessee. The Bryologist, 75 (4), $481-500$.

Sutton, C.S., 1928 : A sketch of the vegetation of the Cradle Mountain, Tasmania, and a census of its plants. Pap. Proc. R. Soc. Tasm., $132-159$.

Weber, W.A., 1969 : Lichenes Exsiccati. Distributed by Univ. of Colorado Fasc., $6 \& 7$, Nos. $201-280$.

, 1971 : Lichenes Exsiccati. Distributed by Univ. of Colorado. Fasc. $8 \& 9$, Nos. $281-360$

., 1975 : Lichenes Exsiccati. Distributed by Univ. of Colorado. Fasc. $10-12$, Nos. $361-465$.

Weber, W.A. and Wetmore, C.M., 1972 : Catalogue of the lichens of Australia exclusive of Tasmania. Nova Hedwigia, 41, i - 137.

Wetmore, C.M., 1963 : Catalogue of the lichens of Tasmania. Rev. Bry. et Lich., XXXII, FASC. 1-4, $223-264$.

., 1967 : Lichens of the Black Hills of South Dakota and Wyoming. Plbi. Mus. Michiqan State University, Biological Series, 3, (4), $209-364$.

Wilson, F.R.M., 1892 : The Lichens of Victoria, Part 1. Proc. R. Soc. Vict., 5 (2nd Series), 141 - 177.

Yoshimura, I., 1973 : Notes on Gumnoderma meZacarpim Comb. Nov. J. Jap. Bot., 48 (9), $283-288$. 


\begin{tabular}{|c|c|c|c|c|c|}
\hline Lichen & 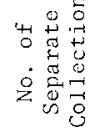 & $\begin{array}{l}\text { Altitude } \\
\text { Range } \\
\text { metres }\end{array}$ & Substrate & Typical llabitat & $\begin{array}{l}\text { Typical Collection } \\
\text { Locality } \\
\text { (Division of State } \\
\text { in Brackets) }\end{array}$ \\
\hline Acarospora schleicheri (Ach.) Mass. & 5 & $30-460$ & $\begin{array}{l}\text { sands tone, dolerite, } \\
\text { granite }\end{array}$ & $\begin{array}{l}\text { exposed rock outcrops } \\
\text { coastal \& inland }\end{array}$ & Spring Bay (E) \\
\hline Apthonia cinnabarina (D.C.) Wallr. & 1 & 600 & Metalenca sp. & MeZateuca groves & Lemonthyme (N.W.) \\
\hline Aspicitia alpina (Sommerf.) Arn. & 3 & $760-1250$ & dolerite, quartzite & $\begin{array}{l}\text { exposed mountain } \\
\text { ridges }\end{array}$ & Mt. Snowy (S) \\
\hline Buellia pulchella Tuckerm. & 11 & $760-1280$ & dolerite \& moss & $\begin{array}{l}\text { crevices in trountain } \\
\text { boulders }\end{array}$ & Mt. Wellington (S) \\
\hline Cladonici bacillaris (Ach.) Nyl. & 5 & $400-1070$ & doleritic soil & mountain moorland & $\begin{array}{l}\text { Moors nr. Great } \\
\text { Lake (C) }\end{array}$ \\
\hline Cladonia didyma (Fee') Vain. & 1 & 60 & button grass soi I & button grass plains & Picton Plains (S.W.) \\
\hline Cladonia ecmocyna (Ach.) Nyl. & 1 & 1520 & doleritic soi 1 & $\begin{array}{l}\text { exposed mountain } \\
\text { outcrops }\end{array}$ & itt. ossa $(C)$ \\
\hline Cladonia subüigitata Vain. & 31 & $60-1520$ & $\begin{array}{l}\text { soil and rotting } \\
\text { logs }\end{array}$ & $\begin{array}{l}\text { sheltered forests \& } \\
\text { open moorlands }\end{array}$ & Mt. Mawson (C) \\
\hline Coccotrena curceubitula Müli. Arg. & 24 & $60-1280$ & $\begin{array}{l}\text { soil and rotting } \\
\operatorname{logs}\end{array}$ & $\begin{array}{l}\text { mountain bouiders } \\
\text { lowland moors }\end{array}$ & $\begin{array}{l}\text { Cradle PIateau } \\
\text { (N.W.) }\end{array}$ \\
\hline Ephebe frutieosa Henssen & 18 & $120-1200$ & dolerite & $\begin{array}{l}\text { mountain boulders or } \\
\text { rocks in streams }\end{array}$ & $\begin{array}{l}\text { Devil's Gullet } \\
\text { (N.W.) }\end{array}$ \\
\hline Lecanora badia (Hofftm.) Ach. & 1 & 900 & quartzite & mountain boulders & Mt. Arrowsmith $(W)$ \\
\hline Leconora ḃ Lando Nyl. & 12 & $60-1040$ & $\begin{array}{l}\text { dead wood and } \\
\text { rocks }\end{array}$ & $\begin{array}{l}\text { rocks and logs in } \\
\text { light timber }\end{array}$ & Mit. Faulkner (S) \\
\hline Lecanora cenisea Ach. & 9 & $2-180$ & $\begin{array}{l}\text { dolerite, sands tone } \\
\text { limestone, soil }\end{array}$ & maritime rocks & Spring Beach (E) \\
\hline Lecidea dickeoniz (J.F. Gmel.) Ach. & 5 & $460-1520$ & dolerite & mountain boulders & Ben Lomond (N.E.) \\
\hline Lecidea friesii Ach. in Libjelbl. & 9 & $460-1220$ & $\begin{array}{l}\text { burnt and dead } \\
\text { Eucalyptus logs }\end{array}$ & $\begin{array}{l}\text { burnt areas on } \\
\text { mountain sides }\end{array}$ & $\begin{array}{l}\text { Sth. Wellingtori } \\
\text { Gap (S) }\end{array}$ \\
\hline $\begin{array}{l}\text { Meregazzia aeneofusca (Mü11. Arg.) } \\
\text { Sant. }\end{array}$ & 20 & $460-1340$ & $\begin{array}{l}\text { dolerite, granite } \\
\text { quartzite }\end{array}$ & mountain boulders & Mt. Marion (S) \\
\hline Nenegazzia globulifera Sant. & 11 & $670-1220$ & $\begin{array}{l}\text { Nothofagus, Richea, } \\
\text { Telopea Sp. }\end{array}$ & Mothofagus forests & Nt. Rufus (C) \\
\hline Veiropocon eilictus v. copillaris & 4 & $1040-1220$ & dolerite & rocky outcrops on & Lake Augusta (C) \\
\hline
\end{tabular}


TABLE 1

(Continued)

NEW LICHEN RECORDS FOR TASMANIA (RECENT COLLECTIONS)

Lichen

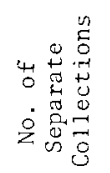

Parmelia cheeliz Gyel.

Parme lia dichotoma Muil1. Arg.

Parmelia furcata Müll. Arg.

Parmelia scabrosa Tayl.

Parmelia subcaperata Kremp.

Parmelia taractica Tayl.

ELacopsis trachyderma $v$.

elavifera (M. Lamb.) James

Eseudocyphellaria amphisticta

$$
\text { (Knight) }
$$

Fseudocyphellania australiensis H. Magn.

Pseudocyphezlaria intricata (Del.) Vain.

Psoroma contextum Stirt.

Esoroma paleacewn (Fr.) Nyl.

Siphuza fragizis (Hook. et Tayl.)

Murray in ed.

\section{Altitude}

Range

metres

Substrate

12

$80-1220$

granite, quartzite

sandstone, dolerite

3- 550

granite, sandstone

dolerite

$9 \quad 3-600$

granite, sandstone

dolerite

15

3-1160

dolerite, basalt

sandstone, granite

15

$6-600$

dolerite, sandstone

granite

$15-460$

mudstone, sandstone

dolerite, granite

soil

$4300-600$ mine spil

9

66

$300-1040$

MeLaleuca and

Nothofaqus sp.

$6-900$

dolerite, sandstone

granite, quartzite

$180-900$

Acacia and

Nothofagus $\mathrm{Sp}$.

$21 \quad 300-1160$

MeZaleuca Sp.

dead wood

19

$460-1220$

soil and dead wood

$800-1620$ doleritic soil, moss cushion plants
Typical Habitat

Typical Collection Locality

(Division of State

in Brackets)

open mountain slopes

Mit. Amos (E)

dry sclerophyll areas

Mt. Amos (E)

dry sclerophyll areas

Lake Leake (C)

open forests

Tab1e Mt. (C)

Grass Tree Hill (S)

East Risdon (S)

Eucalyptus forest

rocks in light

Eucaliptus forest

recently exposed rock

Mt. Bischoff (N.W.)

damp sheltered

forests

rocks in light

Eucalyptus forest

damp sheltered

forest

damp sheltered

mourtain moors and

light forests

mountain moors

Mt. Picton (S.W.)

Waterfall Bay (S)

Western Bluff

(N.W.)

Wedge River (S.W.)

Lake Dobson

Cradle Plateau (C)

Notes on Table 1. - Divisions of State are - $E=$ East; $C=$ Central; $S=$ South; N.W. = North West; 
TABLE 2

NEW TASMANIAN LICHEN RECORDS FROM WEYMOUTH COLLECTIONS

Lichen

Arthonia complanata Feé

Arthonia propinqua Nyl.

Arthonia radiata (Pers.) Ach.

Arthopyrenia gemelzipara (Kn. Mill. Arg.

Arthothelizm infugaatum Müll. Arg.

Arthothelium interveniens (Ny1.) Zahlbr.

Bacidia atrogrisea (De. apud Hepp.) Korh.

Bacidia buchani (Kn.) Hellb.

Bacidia millegrana (Tayl.) Zahlbr.

Blastenia endochromoides (Nyl.) Müll. Arg.

Gatizlaria melaclinea (Nyl.) Zahlbr.

Graphina platycarpa Zahlbr.

Graphis scripta Ach.

Lecanora atra (Huds.) Ach.

Lecanora cancriformis (Hoffm.) Vain.

Lecanora glaucoflanens Müll. Arg.

Lecidea contigua (Hoffm.) Fries

Lecidea meZancheima Tuck.

Lecidea leptoloma Müll. Arg.

Lecidea pminosula Müll. Arg.

Leptogizom burgessiz (Linn. apud Mum.) Mont.

apud Webb

Leptogizm molaccunum Vain.

opegrapha atra Pers.

opegrapha varia Pers.

Opegrapha vulgata (Ach.) Ach.

Parmelia concotor Sprgl.

Parmelia prolixa v. Bubprolixa (Kremp.) Zahlbr. 784

Pamentaria plrenastroides (kin.) Mili. Arg. 934

Pertusamia atpina Hepp. apud Ahles

Pertasaria cupritaris Kn.

Perpusaria laziós kn.

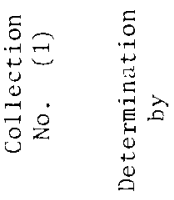

Collection Locality

723

730

643

834

967

968

853

886

286

106

$286(C)$

606

908

162

309

264

340

D.

729

674

960

957

H.

764

685

70

953

831

945

Catamaran

Longley

Brisbane St., Hobart

St. Patrick's Head

Recherche Bay

Hobart

St. Mary's Pass

Lenah Valley

nr. Geeveston

"Huon Valley
Wellard Ck, Tasman Pen.

Wellard Ck, Tasman Pen.

"Cárnarvon", Tasman Pen.

Kermandie, nr. Geeveston

Strickland Ave, nr. Hobart

Strickland Ave, nr. Hobart

Strickland Ave, nr. Hobart

McR obies Gulley, nr. Hohart

Millhouse's Falls, Huon Rd,nr.Hobt.

St.Crispin's Well, Mt. Wellington

Millhouse's Falls, Huon Rd.nr.Hobt.

Wellard Ck, Tasman Pen.

Guy Fawke's Rivulet, nr. Hobart

"Springfield", nr. Johnny's Ck.

Hobart Ri:ulet, Hobart

Wellard Ck, Tasman Pen.

Watchorn's Hill, nr. Hobart

"Carnarvon", Tasman Pen.

"Carnarvon", Tasman Pen.

"Carnarvon", Tasman Pen.

Sugar Loaf Hill, nr. Lindisfarne 


\section{G.C. Bratt and J.A. Cashin}

TABLE 2

(Continued)

NEW TASMANIAN LICHEN RECORDS FROM WEYMOUTH COLLECTIONS

Lichen

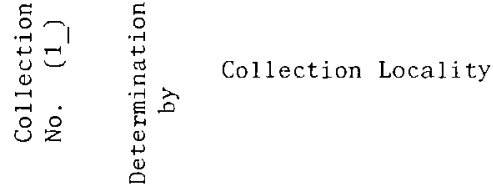

Pertusaria multipunctata (Turn.) Ny1.

$928 \quad 3 \quad$ Blue Tier

Pertusaria nitidula Müll. Arg.

862 " Strickland Ave, nr. Hobart

Phlyctelza sordida (Kn.) Müll. Arg.

969 " Strickland Ave, nr. Hobart

Phlyctella wilsoniz Mül1. Arg.

1692 Guy Fawke's Rivulet, nr. Hobart

Pyrenula mamiliana (Ach.) Trevis

$707 \quad$ "Newmans Ck. Tasman Pen.

Pyremila nitida f.elaeodes (Leight.) A.L. Sm, 70

Romalina polzinaria v. rupestris Flk. apud Schaer

The Lotrema decorticans Müll. Arg.

$897 \quad 3$ Ramsgate

7252 Wellard Ck. Tasman Pen.

Notes on Table 2. (1) Collection numbers and letters used by W.A. Weymouth Identifications by (2) F.R.M. Wilson

(3) R. Paulson

(4) A. Jatta 
TABLE 3

NEW TASMANIAN LICHEN RECORDS FROM PUBLICATIONS

Lichen

Agyrophora subglabra (Ny1.) Liano

Anzia wilsonii Raes.

Candellariezza vitelzina (Ny1.) A.L. Sm.

Catizlania nimosa (Mü11. Arg.) Zah1br.

Cetraria scutata (wulf.) Poetsch

Cladia fuziginosa R. Filson.

CZadia sulzivarii (Mül1. Arg.) W. Martin

Cladonia "delicatuza"(1)

Czadonia "zeptophyzza"(1)

Colzema coccophomm Tuck.

Coltema crispum (Huds.) Wigg

Collema durietait Dege1.

Colzema glaucophthalmum v. glaucophtalmum Dege1.

Collema Zaeve v senecionis (F. Wils.) Degel

cozzema subconveniens Nyl.

Colzema subflaceidum Degel.

Gymnoderma meZacarpum Yoshimura

Lichina tasmanica Henssen.

ochrozechia parezza (L.) Mass.

Parme lia emmpens Kurok.

Parmelia notata Kurok.

Ramalina geniculata v. compacta Mü11. Arg.

Ramalodium suceulentum Nyl, Ex. Cromb.

Rhizocarpon geographicum (L.) D.C.

Teloschistes spinosus (Hook. f. et Tayl.) J. Murray.

Tetoschistes spinosus $f$. subteres Filson.
Reference

Blackman et a1.1974

Bratt et al. 1976

Cribb $1954^{(2)}$

Cribb $1954^{(2)}$

Weber 1975

Filson 1970

Martin 1965 (b)

Sut ton $1928^{(3)}$

Sutton $1928^{(3)}$

Degelius 1974

Degelius 1974

Degelius 1974

Degelius 1974

Degelius 1974

Degelius 1974

Degelius 1974

Yoshimura 1973

Henssen 1969

Cribb 1954

Kurokawa 1969

Kurokawa et al. 1971

Weber 1971

Weber 1975

Sutton 1928

Fi1son 1969

Filson 1969 
G.C. Bratt and J.A. Cashin

TABLE 3

(Continued)

NEW TASMANIAN LICHEN RECORDS FROM PUBLICATIONS

Lichen

Imbilicaria polyphyzza (1.) Baumg.

Usnea capizlacea Motyka

usnea flexitis stirt.

Verrucaria mierosporoides Nyl.

Xanthoria ectanea (Ach.) Raes ex Filson.
Reference

Blackman et al 1974

Weber 1969 \& 1975

Weber 1969

Cribb 1954

Filson 1969

Notes on Table 3. (1) These epithets have not been traced to date

(2) Identification by P. Bibby

(3) Identifications probably by L. Rodway 
TABLE 4

NUMERICAL COMPARISON OF FLORAS

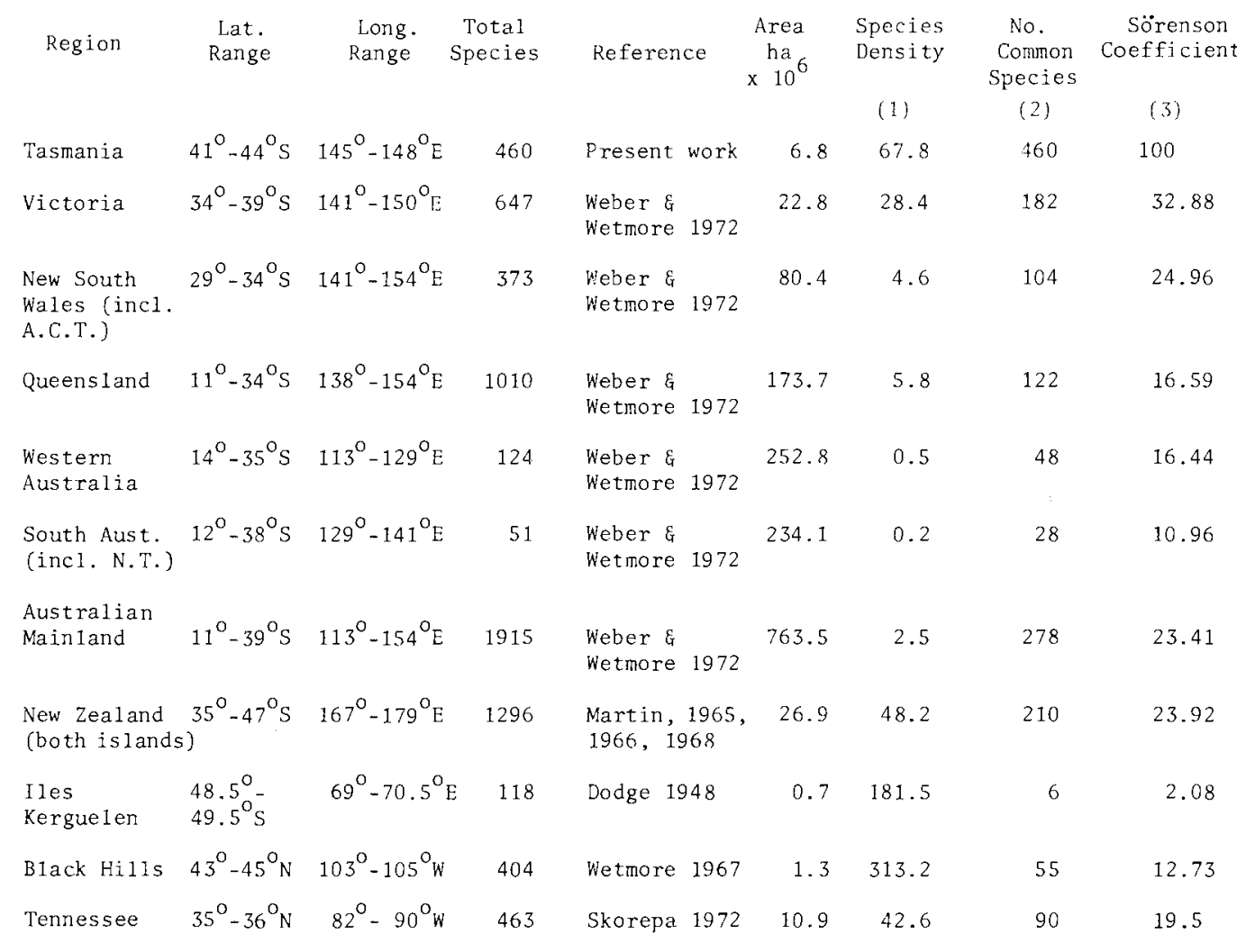

1. Species Density $=$ number of species $/ 10^{6}$ hectare

2. No. of species in common with those in Tasmania

3. Sörenson Coefficient $=\frac{200 \times \text { number of species in common }}{s p \text {. in Area } A+s p \text { in Area } \bar{B}}$ 